\title{
Measurement of Strain-Amplitude Dependent Internal Friction with Torsion Pendulum and Vibration Reed Methods
}

\author{
Qianfeng Fang ${ }^{a, b * *}$, Xianping Wang ${ }^{a, b}$, Zhong Zhuang ${ }^{a}$ \\ ${ }^{a}$ Key Laboratory of Materials Physics, Institute of Solid State Physics, Chinese Academy of Sciences, \\ Hefei, PR China \\ ${ }^{b}$ Suzhou Damping Instruments Company limited, Suzhou, PR China
}

Received: September 23, 2017; Accepted: April 18, 2018

\begin{abstract}
In the traditional standard of internal friction measurement the torsion pendulum and vibration reed were preferentially recommended where the issue of strain amplitude dependence of internal friction was not concerned. To more precisely measure the internal friction and modulus at various strain amplitude, in this paper the strain amplitude dependence of internal friction and modulus is considered by analyzing the stress distribution in the sample in different internal friction measurement methods. The formulas for the measurement of internal friction and modulus versus strain-amplitude are obtained by re-deriving the principal equations for internal friction and modulus measurements by torsion pendulum and vibration reed methods from the basic definition of internal friction. This provides a new standard for precise measurement of internal friction at different strain-amplitude in the cases of high strain-amplitude excitation or high damping materials where amplitude dependent effects always appear.
\end{abstract}

Keywords: Amplitude dependent internal friction, ADIF, Ke's pendulum, Vibration reed.

\section{Introduction}

The internal friction technique or mechanical spectroscopy is very sensitive to the microstructure evolution and various defects in solid state materials ${ }^{1}$, and can be applied in many research fields of condensed matter physics and materials sciences to provide information that is not easy to be obtained by other techniques. Therefore, the accurate measurement of internal friction is of great importance. Nowadays, the most widely used vibration modes to measure internal friction are torsion as in Ke's pendulum ${ }^{2}$ and flexure as in vibration reed $^{3}$. However, the traditional standard for internal friction measurement based on these two modes did not consider the strain amplitude dependence of internal friction. If the internal friction as well as the elastic modulus is strongly dependent upon the strain (or stress) amplitude of the vibrating samples, the traditional standard for internal friction measurement is not capable to obtain the accurate values of the strain amplitude dependent internal friction (ADIF).

The phenomenon of strain amplitude dependence of internal friction was firstly reported by $\mathrm{Read}$ in $\mathrm{Cu}^{4}$. Since then, the amplitude dependent internal friction was reported in many cases such as dislocation relaxation and boundary relaxation, especially in high damping materials ${ }^{5}$, and larger strain amplitude excitation ${ }^{6}$. Some theoretical models on the origin of ADIF were also suggested ${ }^{7}$, including the famous G-L model ${ }^{8}$. However, few efforts were done on how to accurately measure the internal friction when it is amplitude dependent. In this paper, The formulas for the measurement of internal friction and modulus versus strain-amplitude are obtained by re-deriving the principal equations for internal friction and modulus measurements by torsion pendulum and vibration reed methods from the basic definition of internal friction, which would provides a new standard for precise measurement of internal friction at different strain-amplitude.

\section{The Case of Ke's Pendulum}

In the case of Ke's pendulum, the strain amplitude of the vibrating sample is not uniformly distributed, as shown in the left part of Fig.1. Taking a cylindrical sample with radius of $a$ and a length of $L$ as an example, if the sample was twisted by an angle of $\phi$, the strain on the sample surface is the maximum and equals to $\varepsilon_{0}=\frac{a}{L} \phi$, while the strain in the center of sample is zero. Therefore, the strain increases linearly from the center to the surface of the sample in the radial direction. However, the strain is uniform in the length direction. At a site away from the center with a distance of $r$, the strain is $\varepsilon=\frac{r}{L} \phi=\frac{r}{a} \varepsilon_{0}(r=0 \sim a)$. Hence, if the internal friction and shear modulus are strain amplitude dependent, that is, they are functions of strain amplitude: $Q^{-1}(\varepsilon)$ and $G(\varepsilon)$, the internal friction and shear modulus measured according to the traditional standard are not the values at strain amplitude $\varepsilon_{0}: Q^{-1}\left(\varepsilon_{0}\right)$ and $G\left(\varepsilon_{0}\right)$, instead they are the average values over the strain amplitude between 0 and $\varepsilon_{0}$, owing to the nonuniform distribution of strain in the sample. 


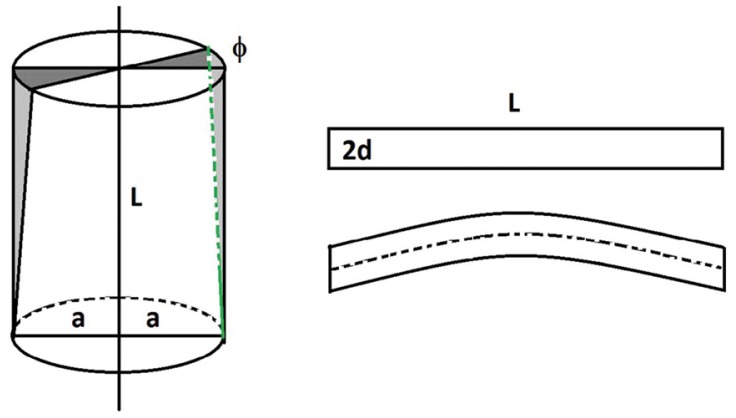

Figure 1. Schematic distribution of the strain in samples for Ke's pendulum (left) and vibration reed (right).

One denotes the internal friction and shear modulus measured at a surface strain amplitude of $\varepsilon_{0}$ as $Q^{-1}{ }_{m}\left(\varepsilon_{0}\right)$ and $G_{m}\left(\varepsilon_{0}\right)$, respectively, which are weighted averages of the intrinsic values of samples $\left(Q^{-1}(\varepsilon)\right.$ and $\left.G(\varepsilon)\right)$ over the strain amplitude range from 0 to $\varepsilon_{0}$. To obtain the relationship between the measured values and the intrinsic values, one can start from the definition of internal friction:

$$
Q^{-1}=\frac{1}{2 \pi} \frac{\Delta W}{W}
$$

where $\Delta W$ and $W$ are the dissipated energy and maximum stored energy of the sample in one vibration cycle (phase angle of $2 \pi$ ), and the $W$ can be written as

$$
W=\frac{V}{2} G(\varepsilon) \varepsilon^{2}
$$

where $V=L \pi a^{2}$ is the volume and $G(\varepsilon)$ is the shear modulus of the sample.

Then, one can choose a thin layer of sample with the inner and outer radius as $r$ and $r+d r$, respectively, the strain amplitude of which can be considered uniform as $\varepsilon=\frac{r}{a} \varepsilon_{0}$. The volume of this thin layer of sample is $d V=2 \pi L r d r$, and the stored elastic energy can be written as $d W$ :

$$
d W=d V \frac{1}{2} G(\varepsilon) \varepsilon^{2}=\frac{\pi \phi^{2}}{L} G(\varepsilon) r^{3} d r
$$

Therefore, the stored elastic energy of the total sample is:

$$
W=L \pi a^{2} \varepsilon_{0}^{2} \int_{0}^{1} G\left(x \varepsilon_{0}\right) x^{3} d x
$$

where the relations of $x=r / a$ and $\varepsilon_{0}=\frac{a}{L} \phi$ were used.

In the traditional standard, the modulus is assumed to be independent of strain amplitude. Under this assumption, equation (4) can be calculated as:

$$
W=\frac{1}{4} L \pi a^{2} \varepsilon_{0}^{2} G_{m}\left(\varepsilon_{0}\right)
$$

where $G_{m}\left(\varepsilon_{0}\right)$ is the measured shear modulus according to the traditional standard when the surface strain amplitude of sample is $\varepsilon_{0}$. By comparing equations (4) and (5), one can obtain the relationship between the measured and intrinsic value of the shear modulus

$$
G_{m}\left(\varepsilon_{0}\right)=4 \int_{0}^{1} G\left(x \varepsilon_{0}\right) x^{3} d x
$$

Taking differentiation on the both side of the above equation with $\varepsilon_{0}$, and multiplying a factor of $\varepsilon_{0} / 4$, one has:

$$
\begin{aligned}
& \frac{\varepsilon_{0}}{4} \frac{\partial G_{m}\left(\varepsilon_{0}\right)}{\partial \varepsilon_{0}}=\int_{0}^{1} x^{4} \frac{\partial G\left(x \varepsilon_{0}\right)}{\partial x} d x=\int_{0}^{1} x^{4} d G\left(x \varepsilon_{0}\right)= \\
& G\left(\varepsilon_{0}\right)-4 \int_{0}^{1} x^{3} G\left(x \varepsilon_{0}\right) d x
\end{aligned}
$$

Substituting equation (6) into (7), and after some reformation, one can obtain

$$
G\left(\varepsilon_{0}\right)=G_{m}\left(\varepsilon_{0}\right)+\frac{\varepsilon_{0}}{4} \frac{\partial G_{m}\left(\varepsilon_{0}\right)}{\partial \varepsilon_{0}}
$$

On the other side, the dissipated energy of this thin layer of sample is $d \Delta W$ :

$$
d \Delta W=2 \pi d W Q^{-1}(\varepsilon)=\frac{2 \pi^{2} \phi^{2}}{L} G(\varepsilon) Q^{-1}(\varepsilon) r^{3} d r
$$

Taking $G^{\prime}(\varepsilon)=G(\varepsilon) Q^{-1}(\varepsilon)$, which is also known as dissipation modulus, the dissipated energy of the total sample is:

$\Delta W=\int d \Delta W=2 L \pi^{2} a^{2} \varepsilon_{0}^{2} \int_{0}^{1} x^{3} G^{\prime}\left(x \varepsilon_{0}\right) d x$

Similarly, one can obtain the relationship between the measured and intrinsic value of the dissipation modulus

$$
G^{\prime}\left(\varepsilon_{0}\right)=G_{m}^{\prime}\left(\varepsilon_{0}\right)+\frac{\varepsilon_{0}}{4} \frac{\partial G_{m}^{\prime}\left(\varepsilon_{0}\right)}{\partial \varepsilon_{0}}
$$

According to the definition of internal friction, $Q^{-1}=\frac{G^{\prime}}{G}$, one can obtain:

$$
\begin{gathered}
\gamma\left(\varepsilon_{0}\right)=1+\frac{\varepsilon_{0}}{4} \frac{\partial \ln G_{m}\left(\varepsilon_{0}\right)}{\partial \varepsilon_{0}} \\
G\left(\varepsilon_{0}\right)=G_{m}\left(\varepsilon_{0}\right) \gamma\left(\varepsilon_{0}\right)
\end{gathered}
$$

$$
Q^{-1}\left(\varepsilon_{0}\right)=Q_{m}^{-1}\left(\varepsilon_{0}\right)+\frac{\varepsilon_{0}}{4 \gamma\left(\varepsilon_{0}\right)} \frac{\partial Q_{m}^{-1}\left(\varepsilon_{0}\right)}{\partial \varepsilon_{0}}
$$

where $\gamma$ is a parameter introduced just for clarity.

\section{The Case of Vibration Reed}

In the case of vibration reed, the strain amplitude of the vibrating sample is not uniformly distributed, as shown in the right part of Fig.1. Taking a plate sample with a thickness of $2 d$ and a length of $L$ as an example, if the sample was flexed at one end, the strain on the sample surface is the maximum, denoted as $\varepsilon_{0}$, while the strain in the center of sample is zero. Therefore, the strain increases linearly from the center to the surface of the sample in the thickness direction. However, the strain is uniform in the directions of length and width. At a site away from the center with a distance of $y$, the 
strain is $\varepsilon=\frac{y}{d} \varepsilon_{0}(y=0 \sim d)$. Hence, if the internal friction is strain amplitude dependent, that is, the internal friction and the Young's modulus are functions of amplitude: $Q^{-1}(\varepsilon)$ and $E(\varepsilon)$, the internal friction and the Young's modulus measured according to the traditional standard are not the internal friction and modulus at strain amplitude $\varepsilon_{0}: Q^{-1}\left(\varepsilon_{0}\right)$ and $E\left(\varepsilon_{0}\right)$, instead they are the average values over the strain amplitude between 0 and $\varepsilon_{0}$, owing to the nonuniform distribution of strain in the sample.

For a thin layer of sample with a thickness of dy and a distance y away from the center of sample, its volume is $d V=L b d y$ (where $\mathrm{b}$ is the width of sample), and its stored and dissipated energy are:

$$
d W=d V \frac{1}{2} E(\varepsilon) \varepsilon^{2}=\frac{L d b}{2} E\left(z \varepsilon_{0}\right) z^{2} d z
$$

$$
d \Delta W=2 \pi d W Q^{-1}(\varepsilon)=\pi L d b E\left(z \varepsilon_{0}\right) Q^{-1}\left(z \varepsilon_{0}\right) z^{2} d z
$$

where $z=y / d$.

According to the similar treatment in the case of Ke's pendulum, if one denotes the internal friction and Young's modulus measured at a surface strain amplitude of $\varepsilon_{0}$ as $Q^{-1}{ }_{m}\left(\varepsilon_{0}\right)$ and $E_{m}\left(\varepsilon_{0}\right)$, respectively, their relationship with the intrinsic values $Q^{-1}\left(\varepsilon_{0}\right)$ and $E\left(\varepsilon_{0}\right)$ can be obtained as:

$$
\begin{gathered}
\eta\left(\varepsilon_{0}\right)=1+\frac{\varepsilon_{0}}{3} \frac{\partial \ln E_{m}\left(\varepsilon_{0}\right)}{\partial \varepsilon_{0}} \\
E\left(\varepsilon_{0}\right)=E_{m}\left(\varepsilon_{0}\right) \eta\left(\varepsilon_{0}\right) \\
Q^{-1}\left(\varepsilon_{0}\right)=Q_{m}^{-1}\left(\varepsilon_{0}\right)+\frac{\varepsilon_{0}}{3 \eta\left(\varepsilon_{0}\right)} \frac{\partial Q_{m}^{-1}\left(\varepsilon_{0}\right)}{\partial \varepsilon_{0}}
\end{gathered}
$$

where $\eta$ is a parameter introduced just for clarity.

\section{The New Standard for Measurement of Amplitude Dependent Internal Friction}

From the above analysis, it can be seen that in the case of strain amplitude dependence, the internal friction and modulus measured according to the traditional standard of internal friction measurement are only approximately equal to the intrinsic values of the sample. Furthermore, even the measured values are also different when measured with different methods, for example, Ke's pendulum and vibration reed. From the equations of (12-14) and (17-19), the difference between the intrinsic and measured values is proportional to the multiplication of strain amplitude with derivatives of internal friction or modulus versus strain amplitude. One will quantitatively analyze such difference via two examples.

One example is the pure $\mathrm{Mg}$ measured by Ke's pendulum at room temperature. The measurement mode is forced vibration and the measurement frequency is $1 \mathrm{~Hz}$. The measured internal friction and relative shear modulus versus strain amplitude are shown in Fig. 2 as open symbols. It can be seen that with increasing strain amplitude the internal friction increases and the modulus decreases monotonously. After data processing according to equations (12-14), the intrinsic values of internal friction and modulus are obtained and shown in Fig. 2 as solid symbols. It can be seen that the intrinsic internal friction is obviously larger than the measured one, and the difference becomes larger with increasing strain amplitude. At a strain amplitude of $10^{-3}$, the intrinsic internal friction of the sample is $20 \%$ higher than the measured value. Similarly, the intrinsic modulus is lower than the measured one and the difference becomes larger with increasing strain amplitude. However, the difference between the intrinsic and measured modulus is less than $1 \%$ 。

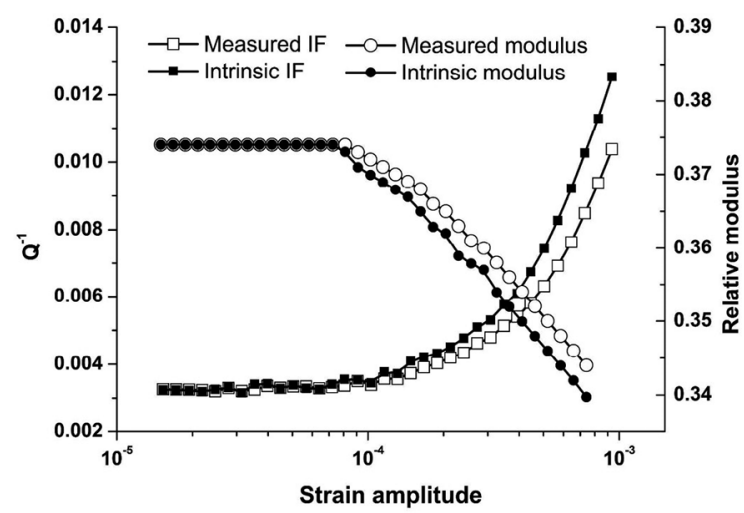

Figure 2. Measured (open) and intrinsic (solid) curves of internal friction and relative shear modulus of pure $\mathrm{Mg}$.

The other example is the $\mathrm{Fe}-\mathrm{Cr}$ based high damping materials measured by Ke's pendulum at room temperature. The measurement mode is again the forced vibration and the measurement frequency is $1 \mathrm{~Hz}$. The measured internal friction and relative shear modulus versus strain amplitude are shown in Fig. 3 as open symbols. It can be seen that with increasing strain amplitude the internal friction increases and then decreases, and a peak appears at the strain amplitude of $3.4 \times 10^{-4}$ with a height of 0.076 . The modulus varies in an opposite trend with the internal friction. After data processing according to equations (12-14), the intrinsic values of internal friction and modulus are obtained and shown in Fig. 3 as solid symbols. It can be seen that the intrinsic internal friction is obviously larger at lower amplitude side but smaller at higher amplitude side than the measured one. At a strain amplitude of $10^{-4}$, the intrinsic internal friction of the sample is $50 \%$ higher than the measured value. As a result, the peak position of the intrinsic internal friction shifts to $2.8 \times 10^{-4}$, and the peak height also increases to 0.081. Similarly, the intrinsic modulus is smaller at lower amplitude side but larger at higher amplitude side than the measured one. However, the difference between the intrinsic and measured modulus is less than $2 \%$ 。 


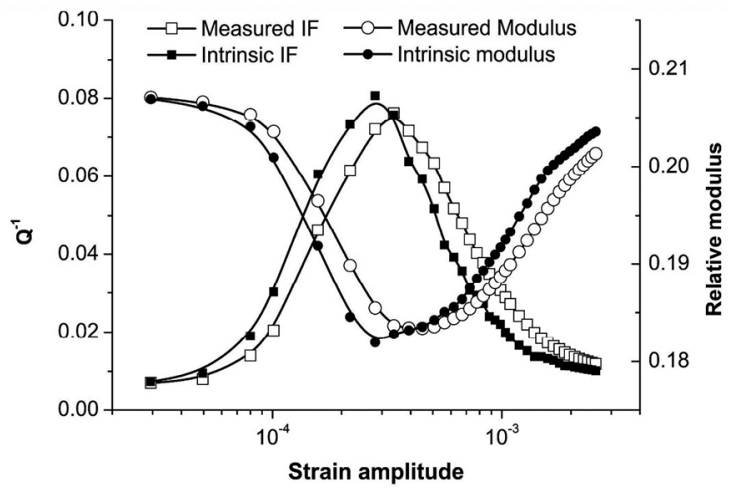

Figure 3. Measured (open) and intrinsic (solid) curves of internal friction and relative shear modulus of $\mathrm{Fe}-\mathrm{Cr}$ based alloys

\section{Conclusions}

From the above discussion, the new standard for measurement of strain amplitude dependent internal friction and modulus can be suggested as follows:

1. Use the forced vibration mode basing on a Ke's pendulum and excite the samples to different maximum strain amplitude on the surface to obtain the curves of internal friction and shear modulus versus strain amplitude: $Q^{-1}{ }_{m}\left(\varepsilon_{0}\right)$ and $G_{m}\left(\varepsilon_{0}\right)$.

2. In the case of low internal friction, use the free decay mode basing on a Ke's pendulum and vibration reed. After exciting the samples to the maximum strain amplitude on the surface, one can obtain the free decay curves of amplitude. Choosing the data on a piece of free decay curve (usually containing 3-5 cycles) one can calculate the values of internal friction and modulus versus average strain amplitude corresponding to that piece of free decay curve by the nonlinear fitting method ${ }^{9}$. Moving the position of this piece curve on the whole free decay curve, one can obtain the curves of internal friction and shear modulus or Young's modulus versus strain amplitude: $Q^{-1}{ }_{m}\left(\varepsilon_{0}\right), G_{m}\left(\varepsilon_{0}\right)$ and $E_{m}\left(\varepsilon_{0}\right)$.
3. Finally, according to the equations (12-14) for Ke's pendulum or (17-19) for vibration reed one can calculate the curves of intrinsic internal friction and intrinsic shear modulus or Young's modulus versus strain amplitude: $Q^{-1}\left(\varepsilon_{0}\right), G\left(\varepsilon_{0}\right)$ and $E\left(\varepsilon_{0}\right)$.

\section{Acknowledgement}

This work was financially supported by the National Natural Science Foundation of China (Grant Nos. 11274305).

\section{References}

1. Fang QF, Wang XP, Wu XB, Lu H. The basic principles and applications of internal friction and mechanical spectroscopy. Wuli. 2011;40(12):786-793. In Chinese.

2. Ke TS. Development of the torsion pendulum and early research on grain boundary relaxation and the cold-work internal friction peak. Journal of Alloys and Compounds. 1994;211/212: 7-15.

3. Li ZS, Fang QF, Veprek S, Li SZ. Evaluation of the internal friction and elastic modulus of the superhard films. Materials Science and Engineering A. 2004;370:186-190.

4. Read TA. Internal friction of single crystals of copper and zinc. Transactions of the American Institute of Mining and Metallurgical Engineers. 1941;143:30-41.

5. Yin FX, Takamori S, Ohsawa Y, Sato A, Kawahara K. The Effects of Static Strain on the Damping Capacity of High Damping Alloys. Materials Transactions. 2002;43(3):466-469.

6. Van Ouytsel K, Fabry A, De Batist R, Schaller R. Determination of the yield strength of nuclear reactor pressure vessel steels by means of amplitude-dependent internal friction. Journal of Nuclear Materials. 2000;279(1):51-56.

7. Fang QF. Theoretical treatment of the nonlinear anelastic internal friction peaks appearing in the cold-worked Al-based solid solutions. Physical Review B. 1997;56(1):12617.

8. Granato AV, Lücke K. Temperature dependence of amplitudedependent dislocation damping. Journal of Applied Physics. 1981;52(12):7136-7142.

9. Fang QF, Wang XP, Zhang GG, Cheng ZJ. Evolution of internal friction and dielectric relaxation peaks in La2Mo2O9-based oxide-ion conductors - assessed by a nonlinear peak fitting method. physica status solidi (a). 2005;202:1041-1047. 